\title{
Subcloning and Nucleotide Sequencing of an ARS Site of Candida maltosa Which Also Functions in Saccharomyces cerevisiae
}

\author{
Shinya KawaI, Chel Won Hwang, Masakazu Sugimoto, \\ Masamichi TAKagi and Keiji Yano \\ Department of Agricultural Chemistry, The University of Tokyo, \\ Bunkyo-ku, Tokyo 113, Japan \\ Received December 1, 1986
}

\begin{abstract}
A DNA region exhibiting ARS activity in Candida maltosa was subcloned from an isolated $3.8 \mathrm{~kb}$ DNA fragment and designated as the TRA region previously [M. Takagi et al., J. Bacteriol., 167, 551 (1986)], which replicates autonomously not only in C. maltosa but also in Saccharomyces cerevisiae. It was found that a DNA fragment of about $200 \mathrm{bp}$ (designated as fragment 2) exhibited ARS activity in both yeasts, and nucleotide sequence analysis of this fragment revealed that it contained five 11 bp-sequences which are homologous to the consensus sequence of ARS sites of $S$. cerevisiae [J. R. Broach et al., Cold Spring Harbor Symp. Quant. Biol., 47, 1167 (1982)] (9 among 11 bp being identical in each).

Plasmids constructed using fragment 2 (pTRA2 and pTRA12) will be useful as cloning vectors, although they showed lower transforming frequencies and lower stabilities in C. maltosa than plasmids containing the TRA region (pTRAl and pTRA11).
\end{abstract}

Candida maltosa is one of the $n$-alkaneassimilating yeasts. When cells of these yeasts are transferred from glucose- to $n$-alkanemedium, various proteins are induced in the endoplasmic reticulum and peroxisome. ${ }^{3,4)} \mathrm{We}$ have been studying the induction mechanisms for these specific proteins in $n$-alkane-grown cells of $C$. maltosa. In a previous paper, it was concluded that the synthesis of at least two major inducible proteins in the endoplasmic reticulum is regulated at the level of transcription. ${ }^{5)}$ Detailed investigations on the molecular mechanisms involved in the regulation of gene expression may require the isolation and structural analysis of the genes encoding these proteins. Host-vector systems should be very useful for this purpose. As C. maltosa does not contain any plasmids, a DNA fragment exhibiting "ARS" activity ("autonomously relicating sequence") is required to construct vectors. ${ }^{6)}$

Abbreviations: kb, kilobase pairs; bp, base pairs.
We have succeeded in isolating an ARS site (a $3.8 \mathrm{~kb}$ fragment designated as the TRA region) from the genome of $C$. maltosa which supports the stable replication of a plasmid containing it in $C$. maltosa cells. ${ }^{1)}$ Using the TRA region and the $L E U 2$ gene of $S$. cerevisiae, we constructed $11 \mathrm{~kb}$ vectors (pTRA1 and pTRA11) for a host, C. maltosa J288 $\left(l e u^{-}\right)$, a mutant derived from C. maltosa IAM12247. During this work, it was found that although neither the replication origin of the $2 \mu \mathrm{m}$ plasmid of $S$. cerevisiae nor the ARS1 site isolated from the genome of $S$. cerevisiae can function as a replication origin in $C$. maltosa, the TRA region can also function as an ARS site in $S$. cerevisiae.

In the present investigation, we subcloned the ARS site from the $3.8 \mathrm{~kb}$ TRA region (i) to analyze the structure of DNA fragment which functions as an ARS site in both C. maltosa and $S$. cerevisiae and (ii) to minimize the size of 
plasmids for host-vector systems in C. maltosa.

\section{MATERIALS AND METHODS}

Strains, plasmids and media. The yeast strains used were C. maltosa $\mathbf{J} 288\left(\text { leu }^{-}\right)^{7}$ ) and $S$. cerevisiae AH22 (a leu2-3 leu2-112 his4-519 can1 gal2) ${ }^{8)}$ The synthetic medium for yeasts (SD) contained $0.67 \%$ Yeast Nitrogen Base W/O amino acids (Difco), 2\% glucose and required supplements. YPD medium $(1 \%$ yeast extract, $2 \%$ Bactopeptone and $2 \%$ glucose) was also used. The bacterial strain used was E. coli JA221 (recA1 leuB6 trpE5 hsdR ${ }^{-}$ lac $Y$ ). It was grown in Luria broth. The plasmid vectors used $^{1)}$ were pTRA 1 and pTRA11 (containing pBR322, the $L E U 2$ gene of $S$. cerevisiae plus the TRA region), pBRTRA (pBR322 plus the TRA region) and pBR-LEU (pBR322 plus the LEU2 gene, see Fig. 2).

$D N A$ isolation, transformation and DNA sequencing. E. coli JA221 was used as the recipient for the ligated DNA. Restriction enzymes, the Klenow fragment and T4 DNA ligase were purchased from Takara Shuzo Co., Ltd. and used under the conditions suggested by the supplier. For quantitative determination of the transformation frequency, each plasmid multiplied in $E$. coli was purified by $\mathrm{CsCl}-\mathrm{EtBr}$ gradient centrifugation. Transformation of yeast cells was performed by the Li-acetate-method. ${ }^{9}{ }^{9}$ The nucleotide sequences were determined by the dideoxy method $^{10)}$ using M13mp18 and M13mp19 (Takara Shuzo Co., Ltd.).

Subcloning of an ARS site of C. maltosa from the TRA region. For the subcloning of the ARS site (see Fig. 1), the TRA region $(3.8 \mathrm{~kb})$ was digested with HindIII and the resultant $0.6 \mathrm{~kb}$ fragment (fragment $\mathrm{H}$ ) was isolated. The latter was digested with Sau3AI and the resulting two fragments, $0.4 \mathrm{~kb}$ (fragment 1 ) and $0.2 \mathrm{~kb}$ (fragment 2), were isolated. These two fragments were separately ligated with pBR-LEU digested with BamHI and HindIII to produce pCS27 and pCS28 (see Fig. 2). To produce pTRA 2 and pTRA 12 containing the Bam HI and Pst I sites of pBR322 to clone DNA fragments, fragment 2 was inserted into the XhoI site of pBR-LEU after the 3'recessed ends produced on digestion with $X h o I$ (for $\mathrm{pBR}$ LEU), and HindIII and Sau3AI (for fragment 2) had been filled by incubation with deoxynucleoside triphosphates and the Klenow fragment. Only the orientation of the ligation of the two blunt-end fragments differed between pTRA2 and pTRA12 (see Fig. 2).

Plasmid stability. To assay the stability of plasmids in $C$. maltosa grown in a non-selective medium, yeast cells carrying plasmids were grown in the SD medium without leucine, and at the stationary phase they were inoculated into the SD medium containing $2 \mathrm{~mm}$ leucine at the inoculation size of $1 \%$. An aliquot was removed at intervals, which was plated onto either the SD agar-medium without leucine or the SD agar-medium containing $2 \mathrm{~mm}$ leucine. Plasmid stability was calculated by dividing the number of colonies on the leucine-minus plate by the number of colonies on the leucine-plus plate. The generation of cells after the inoculation was determined by plating an aliquot and then counting the number of colonies on the YPD plate.

\section{RESULTS}

\section{Subcloning of the ARS site}

In Fig. 1, a restriction map for the TRA region (a Bam $\mathrm{HI}-$ Bam $\mathrm{HI}$ fragment) is shown.
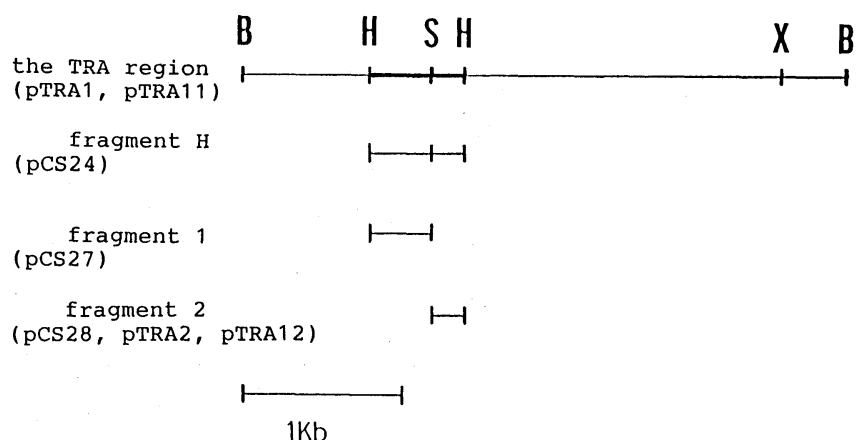

FIG. 1. Restriction Maps of the TRA Region $(3.8 \mathrm{~kb})$ and Subcloned Fragments of It.

The TRA region, cloned as a BamHI-BamHI fragment, was analyzed using restriction enzymes, HindIII and XhoI. The 3 fragments obtained on digestion with HindIII were separately cloned into YEp13. In a transformation experiment, it was found that only the HindIII-HindIII fragment (fragment H) exhibited ARS activity. Fragment $\mathrm{H}$ was digested with $\mathrm{Sau} 3 \mathrm{AI}$, and the resulting two fragments were separately cloned into pBR-LEU to produce pCS27 and pCS28 (Fig. 2). Only fragment 2, i.e., not fragment 1, exhibited ARS activity, which is about $200 \mathrm{bp}$ in size. The names of the plasmids constructed using each of the fragments are given in parantheses. Abbreviations: B, BamiHI; H, HindIII; S, Sau3AI; X, XhoI. 


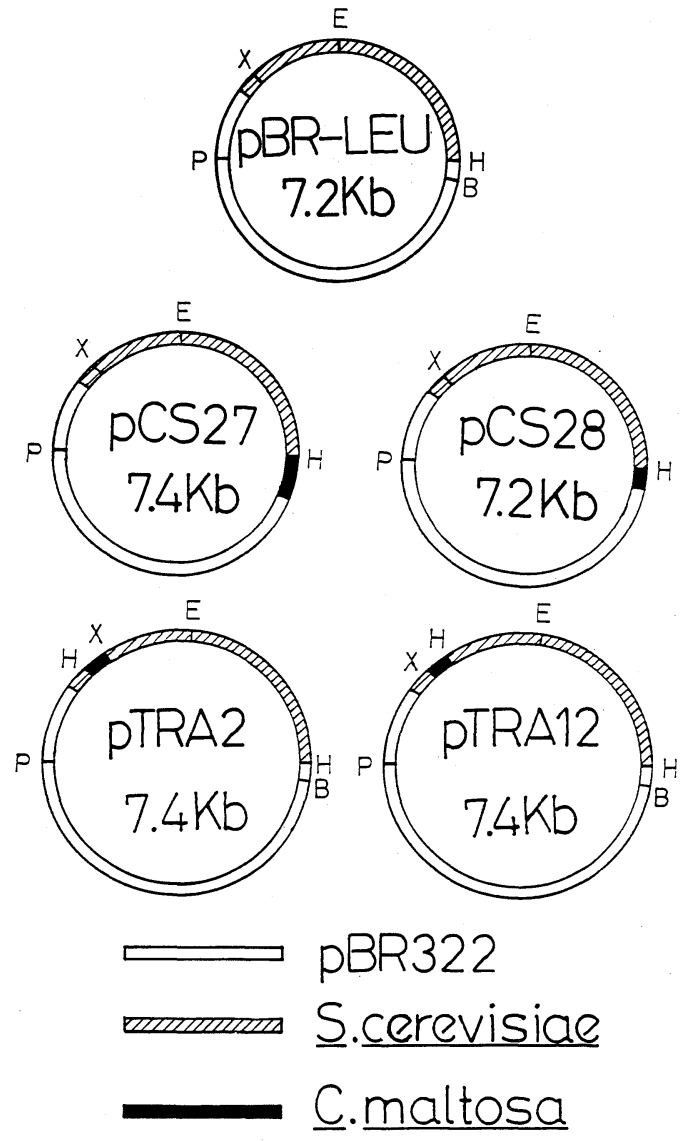

FIG. 2. Plasmids Construction.

To determine which of the two fragments (fragments 1 and 2 in Fig. 1) exhibited ARS activity, each of them was inserted into the BamHI-HindIII site of pBR-LEU (7.2 kb) which consists of pBR322 and the $L E U$ gene of $S$. cerevisiae to produce pCS27 $(7.4 \mathrm{~kb})$ and pCS $28(7.2 \mathrm{~kb})$, respectively. As only pCS28 exhibited ARS activity, fragment 2 was inserted into the XhoI site of pBR-LEU to produce cloning vectors, pTRA2 and pTRA12 (both $7.4 \mathrm{~kb}$ ). Abbreviations: B, BamH1; E, Eco RI; H, HindIII; P, PstI; X, XhoI.

Previously, ${ }^{1)}$ we reported that the XhoI site was located between the left end and the leftside HindIII site of the TRA region, but further analysis of the structure revealed that the $X h o$ I site was very close to the right end, as shown in Fig. 1.

As fragment $\mathrm{H}$ exhibited ARS activity, ${ }^{1)}$ it was digested with Sau3A1, and resulting two fragments (fragments 1 and 2) were ligated with pBR-LEU, which consists of pBR322 and the LEU2 gene of $S$. cerevisiae, to produce pCS27 and pCS28, as shown in Fig. 2. C. maltosa $\mathrm{J} 288\left(\right.$ leu $^{-}$) was transformed with each of these plasmids, and only pCS28 containing fragment 2 gave $\mathrm{Leu}^{+}$transformants.

\section{DNA sequence of fragment 2}

Fragment 2 was inserted between the Bam HI and HindIII sites in the multiple cloning region of either M13mp18 or M13mp19, and both strands were completely sequenced. The results are shown in Fig. 3. Computer analysis was performed to find homologous regions to the consensus sequence of ARSs of $S$. cerevisiae. $^{2)}$ Five 11 bp-sequences were found to be identical in $9 \mathrm{bp}$ to the consensus sequence, which are underlined, and these sequences are summarized in the lower part of Fig. 3.

\section{Construction of cloning vectors with fragment 2}

To construct cloning vectors smaller than the previously described pTRA1 and pTRA11 (each about $11 \mathrm{~kb}$ ), fragment 2 was inserted into the $X$ hoI site of pBR-LEU in two different orientations to produce cloning vectors pTRA2 and pTRA12 (each about $7.4 \mathrm{~kb}$ ), as shown in Fig. 2. They contain the BamHI and Pst I sites for cloning DNA fragments. The frequency of the transformation of these plasmids into $C$. maltosa was about $1 / 3$ of that in the case of pTRA1 and pTRA11.

The stability of these plasmids in C. maltosa grown in a non-selective medium was assayed. As shown in Fig. 4, their stability was lower than that of pTRA1 and pTRA11.

\section{DISCUSSION}

To our present knowledge, the size of a DNA fragment long enough to exhibit ARS activity in yeasts is less than $1 \mathrm{~kb}$. The results presented in this paper indicate that a $196 \mathrm{bp}$ fragment (fragment 2) exhibits ARS activity. As shown in Fig. 3, it has five 11 bp-sequences homologous to the consensus sequence of ARSs of $S$. cerevisiae. At present, it is hard to say which of them are essential or how they 


\begin{tabular}{|c|c|c|c|c|c|}
\hline 10 & 20 & 30 & 40 & 50 & 60 \\
\hline GATCAGTTGT & ATTTAAGTTG & ACAATTTGGT & TCACTAAATT & TAATTAGTTA & GTTGTAAGTC \\
\hline CTAGTCAACA & TAAATTCAAC & TGTTAAACCA & AGTGATTTAA & ATTAATCAAT & CAACATTCAG \\
\hline 70 & 80 & 90 & 100 & 110 & 120 \\
\hline AGTTTTGAAA & ATTTTAGTCA & AATTTTATTA & CCAATTTTTT & ATGTACACTT & AGCAAAAATT \\
\hline TCAAAACTTT & TAAAATCAGT & TTAAAATAAT & GGTTAAAAAA & TACATGTGAA & TCGTTTTTAA \\
\hline 130 & 140 & 150 & 160 & 170 & 180 \\
\hline GAAAGCACTT & TTATACAGTA & GTTACAACGA & GCATACGTGG & TAGTAATGGA & TAATCTATGT \\
\hline CTTTCGTGAA & AATATGTCAT & CAATGTTGCT & CGTATGCACC & ATCATTACCT & ATTAGATACA \\
\hline 190 & 200 & & & & \\
\hline GATATATGAT & AAGCTT & & & & \\
\hline CTATATACTA & TTCGAA & & & & \\
\hline
\end{tabular}

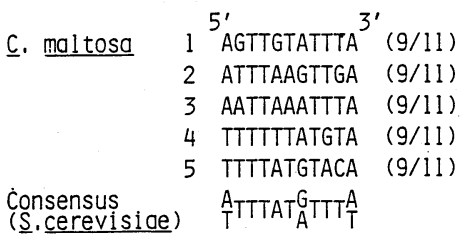

FIG. 3. Nucleotide Sequencing of Fragment 2.

Fragment 2 was sequenced and the sequence is shown from the Sau3A1 site to the HindIII site (see Fig. 1). DNA sequences homologous to the consensus sequence of ARSs of S. cerevisiae (more than $80 \%$ homology) were searched for using a computer, and five sequences were found, which are underlined. Arrowheads indicate the direction of the detected sequence. The 5 homologous sequences are summarized in the lower part, together with the consensus sequence of ARSs of $S$. cerevisiae.

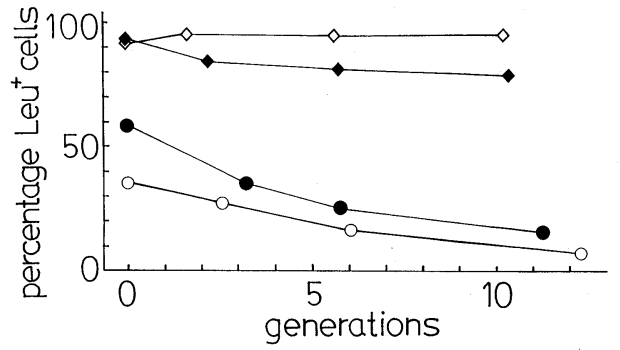

FIG. 4. Stability of pTRA2 and pTRA12 in Comparison to That of pTRA1 and pTRA11 in C. maltosa Grown in a Non-selective Medium.

Cells of $C$. maltosa carrying one of the four plasmids, pTRA1 $(\diamond), \operatorname{pTRA} 11(\diamond), \operatorname{pTRA} 2(\bullet)$ or pTRA12 $(\bigcirc)$, were grown in a non-selective medium. An aliquot was removed at intervals and plated onto plates to determine the total cell number (generations) and the percentage of plasmid-carrying $\mathrm{Leu}^{+}$cells. The averages for two independent experiments are presented.

cooperate for the appearance of ARS activity.

It is expected that the smaller the size of a vector, the lower the number of sites for restriction enzymes is, and thus the greater is the number of restriction enzymes we can use to handle cloned DNA in the vector. The TRA region is $3.8 \mathrm{~kb}$, and pTRA 1 and pTRA 11 are $11 \mathrm{~kb}^{1)}$ in size. Although these plasmids may not be too large for cloning DNA fragments, it might be desirable to improve them by decreasing their size. So, we have constructed two vectors (pTRA2 and pTRA12) using fragment 2 . They can be utilized as cloning vectors in C. maltosa, especially for the subcloning and isolation of DNA fragments using various kinds of restriction enzymes for which there are no cutting sites in these plasmids. It is quite easy to find such kinds of suitable restriction enzymes, since the sequences are now known in all the parts of these plasmids (pBR322, ${ }^{11}$ ) the LEU2 gene ${ }^{12)}$ and fragment 2).

The low frequency of transformation by these plasmids may not be so disadvantageous for their use in the subcloning and isolation of already cloned DNA fragments. Furthermore, 
their low stability in cells grown in a nonselective medium may not be a serious problem in these experiments. These plasmids have the advantage of that they also transform $S$. cerevisiae, although the frequency of transformation is low and the colony sizes of the transformants are small.

It is suggested that during the subcloning the ARS site from the TRA region to fragment 2, some DNA regions were lost, which support efficient transformation and high stability in $C$. maltosa and $S$. cerevisiae. Nevertheless, fragment 2 has an essential DNA region that is functional as an ARS sequence not only in $C$. maltosa but also in $S$. cerevisiae.

\section{REFERENCES}

1) M. Takagi, S. Kawai, M. C. Chang, I. Shibuya and K. Yano, J. Bacteriol., 167, 551 (1986).
2) J. R. Broach, Y.-Y. Li, J. Feldman, M. Jayaram, J. Abraham, K. A. Nasmyth and J. B. Hicks, Cold Spring Harbor Symp. Quant. Biol., 47, 1167 (1982).

3) M. Takagi, K. Moriya and K. Yano, Cell. Mol. Biol., 25, 363 (1980).

4) M. Takagi, K. Moriya and K. Yano, Cell. Mol. Biol., 25, 371 (1980).

5) M. Sunairi, K. Watabe, M. Takagi and K. Yano, J. Bacteriol., 160, 1037 (1984).

6) M. Kawamura, M. Takagi and K. Yano, Gene, 24, 157 (1983).

7) M. C. Chang, H. K. Jung, T. Suzuki, M. Takagi and K. Yano, J. Gen. Appl. Microbiol., 30, 489 (1984).

8) A. Hinnen, J. B. Hicks and G. R. Fink, Proc. Natl. Acad. Sci. U.S.A., 75, 1929 (1978).

9) H. Ito, Y. Fukuda, K. Murata and A. Kimura, $J$. Bacteriol., 153, 163 (1983).

10) J. Messing and J. Vieira, Gene, 19, 269 (1982).

11) J. G. Sutcliffe, Cold Spring Harbor Symp. Quant. Biol., 43, 77 (1979).

12) A. Andreadis, Y.-P. Hsu, M. Hermodoson, G. B. Kohlhaw and P. Schimmel, J. Biol. Chem., 259, 8059 (1984) 